\title{
Modélisation de l'hétérogénéité dans les alliages à mémoire de forme
}

\author{
Abdel Ilah Alhamany ${ }^{1, a}$, Ouadi Mohammed Bensalah ${ }^{2}$ et Omar Fassi Fehri ${ }^{2}$ \\ 1 Université Hassan $1^{\mathrm{er}}$, Faculté Sciences Technique Settat Département de physique, BP 577, Maroc \\ 2 Université Mohamed V, Faculté des Sciences, Rabat, Maroc
}

Reçu le 3 juillet 2003, accepté le 5 novembre 2004

\begin{abstract}
Résumé - Le modèle proposé s'inscrit dans le cadre d'une étude sur le comportement micromécanique métallurgique d'un alliage à mémoire de forme $(A M F) \mathrm{Cu}-\mathrm{Zn}-\mathrm{Al}$ durant la transformation martensitique et l'évolution microstructurale. Le modèle montre que la localisation de la déformation plastique après des sollicitations cycliques entraîne des modifications dans les propriétés mécaniques et thermiques. L'originalité du modèle permet de coupler entre les deux transformations de structures en montrant les mécanismes de déformation nécessaires lors des transformations.
\end{abstract}

Mots clés : Transformation martensitique / variante / plasticité / dislocations / contraintes internes / couplage / potentiel thermodynamique métallurgique

\begin{abstract}
Modeling of heterogeneite in shape memory alloys. The proposed model concerns to study the micromechanical metallurgical behavior of shape memory alloys $\mathrm{Cu}-\mathrm{Zn}-\mathrm{Al}$ (SMAs) during the martensitic transformation and microstructure evolution. The proposed model showed that the accumulation of plastic strains after cyclic tests carries of modifications in the mechanical and thermal properties. Also, the model permit to combine with the martensitic transformation and structure change, explaining the necessery mechanisms involved during the deformation.
\end{abstract}

Key words: Martensitic transformation / variant / plasticity dislocations / internal stresses / couplage / metallurgical thermomechanical potential

\section{Introduction}

Les alliages à mémoire de forme $(A M F s)$ présentent des propriétés thermomécaniques dues à l'existence d'une transformation martensitique thermo-élastique qui peut constituer pour ces matériaux le mécanisme de déformation prédominant [1-6]. Tous ces travaux ont décrit des lois reliant les aspects micro-macro de ces alliages sans tenir compte de changement de structure développé au cours de la déformation. La plupart de ces modèles se réfèrent à des états des défauts observés lors de la transformation martensitique ce qui prive des renseignements précis sur l'état d'évolution de la structure après sollicitation cyclique.

Sur des états fatigués, les observations microscopiques réalisées par microscope électronique en transmission $(M E T)$ montrent une distribution hétérogène des dislocations [7-9]. Elles sont formées sous formes des parois

\footnotetext{
a Auteur correspondant : abdouhamani@hotmail.com
}

très allongées ou des bandes cloisonnées dont la densité augmente avec le nombre de cycles $[9,10]$.

Sur les monocristaux des $A M F s$, les dislocations sont regroupées de façon symétrique après un grand nombre de cycles [11], sur des états polycristallins la structure est beaucoup plus complexe, les défauts sont principalement observés dans les grains et entre les joints des grains $[12,13]$. La majorité des dislocations observées au cours des sollicitations glissent dans le plan de famille $\{011\}$ suivant la direction $\vec{u}=[111]$ de vecteur de Burgers $\vec{b}=[001]$. À partir de ce schéma, il nous semble important de modéliser ces phénomènes physiques de la déformation. L'aspect hétérogène peut être classé dans le domaine de métallurgie qui cherche à analyser le comportement micromécanique à partir des définitions des défauts cristallins créés et de leurs interactions.

L'objectif de ce travail est d'établir un modèle permettant de suivre l'évolution de la structure lors de la localisation de la déformation plastique. De réaliser un couplage entre les deux transformations, la transformation 


\section{Nomenclature}

\begin{tabular}{|c|c|}
\hline$A_{\mathrm{s}}:$ & température de début d'austénite \\
\hline$A_{\mathrm{f}}:$ & température de fin d'austénite \\
\hline$B:$ & coefficient de proportionnalité \\
\hline$\vec{b}$ & vecteur de Burgers \\
\hline$\beta_{i}:$ & traction appliquée à la limite de la surface $\Omega$ \\
\hline$C_{i j k l}:$ & module d'élasticité \\
\hline$\Delta G_{\mathrm{ch}}:$ & énergie libre chimique \\
\hline$E_{i j}:$ & déformation macroscopique totale \\
\hline$E_{i j}^{\mathrm{e}}:$ & déformation macroscopique élastique \\
\hline$E_{i j}^{\mathrm{tr}}:$ & déformation macroscopique de transformation \\
\hline$E_{i j}^{\mathrm{p}}:$ & déformation macroscopique plastique \\
\hline$\varepsilon^{e^{e}}:$ & déformation élastique locale \\
\hline$\varepsilon^{\operatorname{tr}}:$ & déformation de transformation \\
\hline$\varepsilon^{\mathrm{p}}:$ & déformation plastique locale \\
\hline$\Sigma_{i j}:$ & contrainte macroscopique appliquée \\
\hline$f^{\mathrm{h}}:$ & fraction volumique de martensite \\
\hline$f_{\mathrm{p}}:$ & fraction volumique de la zone affectée \\
\hline$\varphi:$ & $\begin{array}{l}\text { l'angle entre le plan de glissement et la normale } \\
\text { aux parois }\end{array}$ \\
\hline$g:$ & amplitude de la déformation de transformation \\
\hline$\gamma_{\mathrm{p}}:$ & amplitude de déformation plastique imposée \\
\hline$h:$ & la variante active \\
\hline$I_{k l p q}:$ & tenseur identité d'ordre quatre \\
\hline & distance entre dislocations de la même paroi \\
\hline$M_{i j k l}:$ & tenseur de compliance \\
\hline$M_{\mathrm{s}}:$ & température de début de martensite \\
\hline$M_{\mathrm{f}}:$ & température de fin de martensite \\
\hline$m_{i}^{h}:$ & direction de déplacement de la variante $h$ \\
\hline$m_{i}^{\mathrm{p}}:$ & direction $\perp$ à la paroi des dislocations \\
\hline$\mu:$ & module de cisaillement \\
\hline$\nu:$ & coefficient du Poisson \\
\hline$n_{i}^{h}:$ & normale au plan d'habitat pour la variante $h$ \\
\hline$n_{i}^{\mathrm{p}}:$ & normale à la paroi des dislocations \\
\hline$n_{j}^{*}:$ & la normale à la limite de la surface $\Omega$ \\
\hline$\Omega:$ & domaine de la transformation martensitique \\
\hline$\Omega_{\mathrm{p}}:$ & domaine de l'évolution microstructurale \\
\hline$\psi:$ & potentiel thermodynamique-métallurgique \\
\hline$R_{i j}^{h}:$ & tenseur d'orientation de la variante $h$ active \\
\hline$R_{i j}^{\mathrm{p}}:$ & tenseur d'orientation de la paroi formée \\
\hline$S_{k l p q}$ & tenseur d'Eshelby \\
\hline$\sigma_{i j}:$ & champ de contraintes internes dû à \\
\hline & $\begin{array}{l}\text { l'incompatibilité d'interaction entre les } \\
\text { variantes }\end{array}$ \\
\hline$\sigma_{i j}^{*}:$ & champ de contraintes internes dû à \\
\hline & $\begin{array}{l}\text { l'incompatibilité d'interaction entre variante et } \\
\text { dislocations }\end{array}$ \\
\hline$T:$ & température d'essai \\
\hline$T_{0}:$ & température d'équilibre thermodynamique \\
\hline$\tau_{0}:$ & $\begin{array}{l}\text { contrainte à saturation à amplitude de } \\
\text { déformation plastique nulle }\end{array}$ \\
\hline$\tau_{\mathrm{s}}$ & contrainte à saturation à amplitude \\
\hline & déformation plastique non nulle \\
\hline$V$ & volume initial de l'austénite \\
\hline$V_{\mathrm{a}}:$ & volume de l'austénite libre \\
\hline$V_{\mathrm{M}}^{h}:$ & volume de martensite \\
\hline$V_{\mathrm{p}}:$ & volume transformé en plastique \\
\hline$W_{\text {elm }}:$ & énergie de déformation élastique \\
\hline$W_{\text {ine }}:$ & énergie de déformation inélastique \\
\hline$X:$ & distance entre la variante et la parois formée \\
\hline
\end{tabular}

martensitique et le changement de structure accompagnée après transformation cyclique.

\section{Modélisation}

\subsection{Les éléments de bases}

Le modèle proposé constitue un cadre suffisamment souple et ouvert pour qu'il soit envisagé de l'appliquer à la modélisation qualitative du comportement micromécanique des alliages à mémoire de forme (AMFs).

Considérons un élément de volume représentatif $V$ d'austénite, sous chargement, une partie de volume se transforme en martensite $V_{\mathrm{M}}^{h}$.

$$
V=V_{\mathrm{a}}+V_{\mathrm{M}}^{h}
$$

avec $V_{\mathrm{a}}$ : volume d'austénite non transformé (Fig. 1).

Nous supposons que la déformation macroscopique totale $E_{i j}$ est la somme d'une déformation macroscopique élastique $E_{i j}^{\mathrm{e}}$, d'une déformation macroscopique de transformation $E_{i j}^{\mathrm{tr}}$, et d'une déformation macroscopique plastique $E_{i j}^{\mathrm{p}}$.

$$
E_{i j}=E_{i j}^{\mathrm{e}}+E_{i j}^{\mathrm{tr}}+E_{i j}^{\mathrm{p}}
$$

À partir de ces définitions des déformations discutées ci-dessus, plusieurs remarques peuvent être utilisées :

- Chaque déformation « $E_{i j} »$ peut être obtenue à partir de la moyenne volumique de toutes les déformations locales $\varepsilon_{i j}$ dans un domaine $D$ correspondant :

$$
E_{i j}=\frac{1}{V} \int_{D} \varepsilon_{i j} \mathrm{~d} V
$$

- La collection de toutes les variantes de martensite formées lors du chargement est représentée par un élément de volume $V_{\mathrm{M}}^{h}$.

- Toutes les contraintes satisfont à l'équation d'équilibre dans le domaine $\Omega$

$$
\sigma_{i j, j}=0 \text { dans } \Omega \text { et } \beta_{i}=\Sigma_{i j} n_{j}^{*}
$$

avec; $\beta_{i}$ : traction dans la limite de la surface $\Omega$. $n_{j}^{*}$ : la normale au voisinage de la frontière $\delta \Omega$.

On définit comme élément de base pour cette modélisation, la déformation de transformation associée à la formation d'une variante de martensite $\varepsilon_{i j}^{h}$ donnée par :

$$
\varepsilon_{i j}^{h}=\sum_{h} g R_{i j}^{h}=\frac{g}{2} \sum_{h}\left(n_{i}^{h} m_{j}^{h}+n_{j}^{h} m_{i}^{h}\right)
$$

où $R_{i j}^{h}$ : est le tenseur d'orientation, $g$ est l'amplitude de déplacement dans la direction de transformation, $n^{h}$ et $m^{h}$ sont respectivement la normale au plan d'habitat et la direction de transformation de la variante $h$ (Fig. 2). 


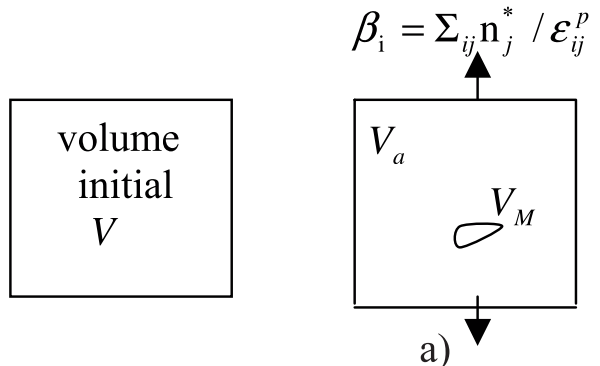

a)

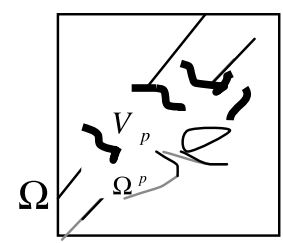

b)

Fig. 1. Schéma d'une microstructure après transformation cyclique. a) Formation de la variante domaine $\Omega$. b) Bandes ou parois de dislocations domaine plastique $\Omega^{\mathrm{p}}$.

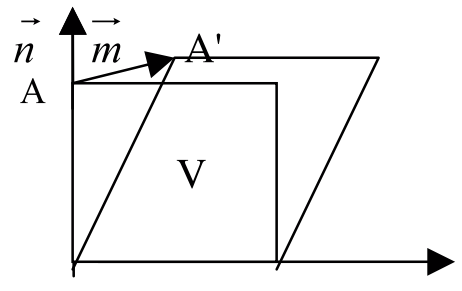

Fig. 2. Représentation d'une déformation de transformation par un élément de volume $\mathrm{V}$.

Pour la déformation de transformation macroscopique on a :

$$
\begin{aligned}
E_{i j}^{\mathrm{tr}} & =\frac{1}{V} \int_{\Omega} \varepsilon_{i j}^{\mathrm{tr}} \mathrm{d} V=\sum_{h} \varepsilon_{i j}^{h} \frac{V_{\mathrm{M}}^{h}}{V}=\sum_{h} \varepsilon_{i j}^{h} f^{h} \\
& =g \sum_{h} R_{i j}^{h} f^{h}
\end{aligned}
$$

avec

$$
f^{h}=\frac{V_{\mathrm{M}}^{h}}{V}
$$

la fraction volumique occupée par la variante $h$ la plus activée.

La déformation plastique $\varepsilon_{i j}^{\mathrm{p}}$ s'écrit :

$$
\varepsilon_{i j}^{\mathrm{p}}=\gamma_{p} R_{i j}^{\mathrm{p}}=\frac{\gamma_{\mathrm{p}}}{2}\left(n_{i}^{\mathrm{p}} m_{j}^{\mathrm{p}}+n_{j}^{\mathrm{p}} m_{i}^{\mathrm{p}}\right)
$$

avec $\gamma_{\mathrm{p}}$ : amplitude de déformation plastique imposée donnée par :

$$
\gamma_{\mathrm{p}}=\frac{1}{V_{\mathrm{p}}} m_{g}^{\mathrm{p}} b_{g}\left\langle S_{\mathrm{p}}\right\rangle
$$

où

$V_{\mathrm{p}}$ : représente le volume de la paroi des dislocations formées,

$m_{g}^{\mathrm{p}}$ : correspond à la direction de vecteur de Burgers $b_{g}$ dans le plan de glissement $g$,

$\left\langle S_{\mathrm{p}}\right\rangle$ la moyenne de l'aire balayée par les dislocations pendant le glissement dans leurs plans.

$R_{i j}^{\mathrm{p}}$ : tenseur d'orientation de la paroi formée.

$$
R_{i j}^{\mathrm{p}}=\frac{1}{2}\left(n_{g_{i}}^{\mathrm{p}} m_{g_{j}}^{\mathrm{p}}+n_{g_{j}}^{\mathrm{p}} m_{g_{i}}^{\mathrm{p}}\right)
$$

$n_{g_{i}}^{\mathrm{p}}$ : normale à la paroi des dislocations.
Suivant l'hypothèse des faibles déformations, la déformation macroscopique plastique $E_{i j}^{\mathrm{p}}$ s'écrit comme la moyenne des déformations plastiques microscopiques :

$$
E^{\mathrm{p}}=\left\langle\varepsilon^{\mathrm{p}}\right\rangle_{V}
$$

\langle\rangle symbole signifie la valeur moyenne d'un champ dans le volume $V$.

La déformation plastique macroscopique est alors :

$$
E^{\mathrm{p}}=\sum_{g} f_{g} \varepsilon_{g}^{\mathrm{p}}
$$

avec $f_{g}=\frac{V_{\mathrm{p}}}{V_{\mathrm{a}}}$ : la fraction volumique de la région transformée plastiquement.

Les fluctuations de la contrainte locale, et de champ de déplacement sont notées par $\widehat{\sigma}, \widehat{u}$.

En tenant compte des conditions de périodicité, les relations entre les composantes micro et macro sont :

$$
\begin{aligned}
\sigma & =\Sigma+\widehat{\sigma} \\
\langle\widehat{\sigma}\rangle_{V} & =0, \quad \Sigma=\langle\sigma\rangle_{V} \\
u(x) & =E+\widehat{u} \\
\varepsilon^{\mathrm{p}} & =E^{\mathrm{p}}+\widehat{\varepsilon}^{\mathrm{p}} \\
\left\langle\widehat{\varepsilon}^{\mathrm{p}}\right\rangle_{V} & =0
\end{aligned}
$$

La déformation plastique totale est donc :

$$
\varepsilon^{\mathrm{p}}=\sum_{g} \varepsilon_{g}^{\mathrm{p}}
$$

où ;

$$
\varepsilon_{g}^{\mathrm{p}}=\varepsilon^{\mathrm{p}} \text { dans } V_{\mathrm{p}} \text { et } \varepsilon_{g}^{\mathrm{p}}=0 \text { dans } V-V_{\mathrm{p}}
$$

\subsection{Formalisme général}

Pour la plupart de ces matériaux, l'origine des changements de structures peut être liée à une énergie de déformation élastique $W_{\text {ela }}[1-6,14]$, d'une énergie inélastique physique fortement dépendante de l'évolution de structure $W_{\text {ine }}$. 
- L'énergie de déformation élastique est la somme de deux termes :

$$
W_{\text {ela }}=\frac{1}{2} \Sigma E^{\mathrm{e}}+\frac{1}{2 V} \int_{\Omega} \sigma \varepsilon^{\mathrm{e}} \mathrm{d} V
$$

le $1^{\text {er }}$ terme correspond à l'énergie de déformation élastique liée aux contraintes macroscopiques $\Sigma$.

$E^{\mathrm{e}}$ : déformation élastique macroscopique liée à la contrainte moyenne macroscopique $\Sigma$ par la relation linéaire d'élasticité :

$$
E^{\mathrm{e}}=M \Sigma
$$

où $M$ : tenseur de compliance.

En utilisant les relations de compatibilité, et en absence des forces surfaciques:

$$
\begin{aligned}
\varepsilon & =\varepsilon^{\mathrm{e}}+\varepsilon^{\mathrm{tr}}+\varepsilon^{\mathrm{p}} \\
\sigma_{j}, n_{j} & =0
\end{aligned}
$$

Dans le domaine $\Omega, \varepsilon_{g}^{\mathrm{p}}=0$, et selon les conditions de compatibilité de la déformation équation (16) le second terme $\left(\frac{1}{2 V} \int_{\Omega} \sigma \varepsilon^{\mathrm{e}} \mathrm{d} V\right)$ devient :

$$
\frac{1}{2 V} \int_{\Omega} \sigma \varepsilon^{\mathrm{e}} \mathrm{d} V=-\frac{1}{2 V} \int_{\Omega} \sigma \varepsilon^{\mathrm{tr}} \mathrm{d} V
$$

$\frac{1}{2 V} \int_{\Omega} \sigma \varepsilon^{\operatorname{tr}} \mathrm{d} V$ : correspond à l'énergie élastique associée aux contraintes internes « $\sigma »$ d'incompatibilité entre l'austénite et la martensite, et l'interaction entre les variantes $h$ et $k$.

En utilisant la solution d'Eshelby Kröner [15], pour une inclusion de forme ellipsoïdale $\sigma$ s'écrit :

$$
\sigma_{i j}=C_{i j k l} \sum_{h}\left(I_{k l p q}-S_{k l p q}^{h}\right)\left(\sum_{k} \varepsilon_{p q}^{k} f^{k}-\varepsilon_{p q}^{h}\right)
$$

où $C_{i j k l}$ : tenseur d'élasticité propre à la martensite, $I$ : tenseur identité d'ordre quatre et $S$ : tenseur, représente la morphologie de la variante.

On substitue les équations (16), (20) dans (15), et après intégration l'énergie de déformation élastique s'écrit :

$$
\begin{aligned}
W_{\text {ela }}= & \underbrace{\frac{1}{2} \sum_{i j} M_{i j k l} \Sigma_{k l}}_{1} \\
& +\underbrace{\frac{1}{2} C_{i j k l} \sum_{h}\left(I_{k l p q}-S_{k l p q}^{h}\right) \varepsilon_{p q}^{h} \varepsilon_{i j}^{h} f^{h}}_{2} \\
& -\underbrace{\frac{1}{2} C_{i j k l} \sum_{h} \sum_{k}\left(I_{k l p q}-S_{k l p q}^{h}\right) \varepsilon_{p q}^{h} \varepsilon_{p q}^{k} f^{h} f^{k}}_{2}
\end{aligned}
$$

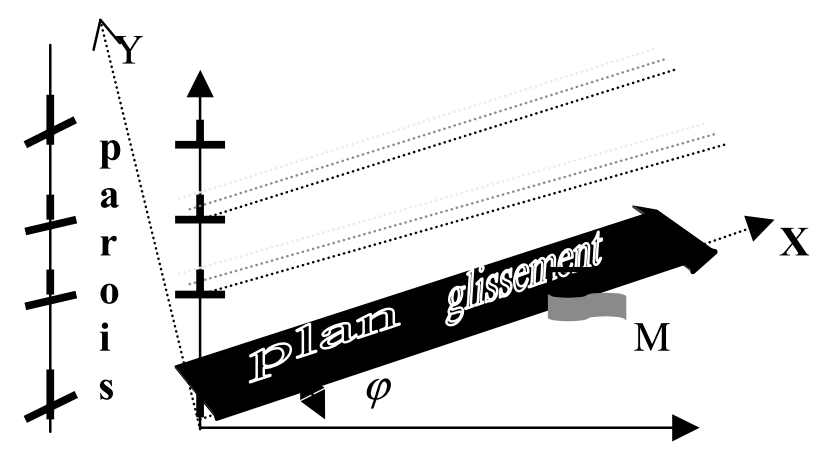

Fig. 3. Arrangement des dislocations après déformation «parois » plan de glissement.

L'énergie de déformation élastique obtenue correspond à une double fonction :

- une fonction linéaire en $f^{h}$ « énergie propre »,

- une fonction quadratique en $f^{h}$ « l'énergie d'interaction entre les deux variantes $»$.

Il reste à modéliser les phénomènes d'écrouissage caractérisant l'évolution de structure et la localisation de la déformation plastique, cette partie rassemble quelques concepts de base de la théorie de plasticité.

Le choix d'une technique approchée à la métallurgie semble cohérente aux résultats microscopiques introduisant des variables caractéristiques des phénomènes observés.

\subsection{Approche métallurgique}

La clé de cette partie de modélisation est de considérer l'aspect hétérogène en présence de la déformation plastique. Pour schématiser les efforts mis en jeu dans les phénomènes que l'on souhaite étudier, il est commode de prendre une forme d'énergie identique à celle présentée dans la première partie.

- L'énergie inélastique s'écrit :

$$
W_{\text {ine }}=-\frac{1}{2 V} \int_{\Omega^{\mathrm{p}}} \sigma^{*} \varepsilon^{\mathrm{p}} \mathrm{d} V
$$

où $\left(\sigma_{i j}^{*}\right)$ champ de contraintes internes due aux incompatibilités de déformation résultant de la répartition hétérogène de dislocations uniforme.

À partir des observations microscopiques [7-11], cette répartition peut être considérée comme un réseau de dislocation vertical rectiligne de longueur infini, formé d'un groupe de dislocations distinctes d'une distance $l$.

On suppose que la variante « point $M »$ est située à une distance $X$ du réseau formé, le plan de glissement $(O, X, Y)$ de la variante est défini par un angle $\varphi$ et par les coordonnées $(X, Y=X \operatorname{tg} \varphi)$ figure 3 .

Par analogie avec le champ de contraintes internes lié à ce type de réseau donné par Li et al. [16], $\sigma_{i j}^{*}$ peut être écrit :

$$
\sigma_{i j}^{*}=A \alpha[\sin 2 \lambda \operatorname{sh} 2 \alpha \sin 2 \varphi+(\operatorname{ch} 2 \alpha \cos 2 \lambda-1) \cos 2 \varphi]
$$


avec

$$
\begin{aligned}
A & =\frac{\mu b}{l(1-\nu)}(\operatorname{ch} 2 \alpha-\cos 2 \lambda)^{-2} \quad \text { et } \\
\alpha & =\frac{\pi X}{l} ; \quad \lambda=\frac{\pi Y}{l}
\end{aligned}
$$

$l$ : correspond à la distance entre deux dislocations de la même paroi (Fig. 3).

Dans le domaine affecté $\Omega^{\mathrm{p}}$, l'énergie inélastique s'écrit donc :

$W_{\text {ine }}=-\frac{A}{2} \alpha[\sin 2 \lambda \operatorname{sh} 2 \alpha \sin 2 \varphi+(\operatorname{ch} 2 \alpha \cos 2 \lambda-1) \cos 2 \varphi]$

$$
\varepsilon_{g}^{\mathrm{p}} \frac{V_{\mathrm{p}}}{V_{\mathrm{a}}} \frac{V_{\mathrm{a}}}{V}
$$

On substitue l'équation (1) dans (25) et en tenant compte de la fraction volumique de la zone affectée, l'énergie devient :

$W_{\text {ine }}=-\frac{A}{2} \alpha[\sin 2 \lambda \operatorname{sh} 2 \alpha \sin 2 \varphi+(\operatorname{ch} 2 \alpha \cos 2 \lambda-1) \cos 2 \varphi]$

$$
\varepsilon_{g}^{\mathrm{p}} f_{g} \sum_{h}\left(1-f^{h}\right)
$$

Cette énergie tient compte d'un nombre important des paramètres physiques microscopiques, de l'interaction entre la variante et les dislocations de la paroi et de la déformation plastique localisée dans le domaine d'austénite libre.

\section{4 Étude spécifique de suivi de l'évolution microstructurale}

L'intérêt de ce type d'approche est de pouvoir introduire à une échelle macroscopique des paramètres physiques de façon à rendre compte des effets mécaniques liés à l'écrouissage.

La loi de comportement globale est définie comme un potentiel thermodynamique-métallurgique $\psi$ associé aux transformations produites (la transformation martensitique et l'évolution de structure) au cours de la déformation.

Notons toutefois que la thermodynamique élémentaire présenté ici sera utilisée sans thermique pour la plupart des phénomènes étudiés mais son cadre est très précieux pour guider et limiter les choix possibles de la modélisation phénoménologique

$$
\psi=\Sigma_{i j} E_{i j}-\left(\Delta G_{\text {ch }}+W_{\text {ela }}+W_{\text {ine }}\right)
$$

On substitue (2) dans (27) $\psi$ devient :

$$
\psi=\Sigma_{i j}\left(E_{i j}^{\mathrm{e}}+E_{i j}^{\mathrm{tr}}+E_{i j}^{\mathrm{p}}\right)-\left(\Delta G_{\mathrm{ch}}+W_{\text {ela }}+W_{\text {ine }}\right)
$$

avec $\Delta G_{\mathrm{ch}}$ énergie libre chimique, fonction de la température $[3,14]$.

$$
\Delta G_{\mathrm{ch}}=B\left(T-T_{0}\right) \sum_{h} f^{h}
$$

avec $B$ : coefficient de proportionalité, $T_{0}$ : température d'équilibre thermodynamique des deux phases (austénite - martensite) [3].

Lors de chargement $T_{0}$ s'écrit :

$$
T_{0}=M_{\mathrm{s}}-\left(M_{\mathrm{f}}-M_{\mathrm{s}}\right) \sum_{h} f^{h}
$$

avec $M_{\mathrm{s}}$ : température de début de martensite, $M_{\mathrm{f}}$ : température de fin de martensite.

Lors de déchargement $T_{0}$ s'écrit :

$$
T_{0}=A_{\mathrm{f}}-\left(A_{\mathrm{S}}-A_{\mathrm{f}}\right) \sum_{h} f^{h}
$$

avec $A_{\mathrm{s}}$ : température de début d'austénite, $A_{\mathrm{f}}$ : température de fin d'austénite.

On substitue les équations (21), (26) et (29) dans l'équation (28) le potentiel $\psi$ s'écrit :

$$
\begin{gathered}
\psi=\frac{1}{2} \Sigma_{i j} M_{i j k l} \Sigma_{k l}-B\left(T-T_{0}\right) \sum_{h} f^{h}+\Sigma_{i j} \sum_{h} \varepsilon_{i j}^{h} f^{h} \\
+\Sigma_{i j} \varepsilon_{g}^{\mathrm{p}} f_{g} \sum_{h}\left(1-f^{h}\right)-\frac{1}{2} C_{i j k l} \sum_{h}\left(I_{k l p q}-S_{k l p q}^{h}\right) \varepsilon_{p q}^{h} \varepsilon_{i j}^{h} f^{h} \\
+\frac{1}{2} C_{i j k l} \sum_{h}\left(I_{k l p q}-S_{k l p q}^{h}\right) \sum_{k} \varepsilon_{p q}^{k} f^{k} \varepsilon_{i j}^{h} f^{h} \\
+\frac{A}{2} \alpha[(\sin 2 \lambda s h 2 \alpha \sin 2 \varphi) \\
+(\operatorname{ch} 2 \alpha \cos 2 \lambda-1) \cos 2 \varphi] \varepsilon_{g}^{\mathrm{p}} f_{g} \sum_{h}\left(1-f^{h}\right)
\end{gathered}
$$

On substitue les équations (5), (11), (30) dans l'équation (32), $\psi$, pour un chargement direct :

$$
\begin{gathered}
\psi=\frac{1}{2} \Sigma_{i j} M_{i j k l} \Sigma_{k l}-B\left[T-\left(M_{\mathrm{s}}-\left(M_{\mathrm{f}}-M_{\mathrm{s}}\right) \sum_{h} f^{h}\right)\right] \\
\times \sum_{h} f^{h}+g \Sigma_{i j} \sum_{h} R_{i j}^{h} f^{h}+\Sigma_{i j} \gamma_{\mathrm{p}} R_{i j}^{\mathrm{p}} f_{g} \sum_{h}\left(1-f^{h}\right) \\
-\frac{g^{2}}{2} C_{i j k l}\left[\sum_{h}\left(I_{k l p q}-S_{k l p q}^{h}\right) R_{p q}^{h} R_{i j}^{h} f^{h}\right. \\
\left.-\sum_{h}\left(I_{k l p q}-S_{k l p q}^{h}\right) \sum_{k}\left(R_{p q}^{k} f^{k}\right) R_{i j}^{h} f^{h}\right] \\
+\frac{A}{2} \alpha[(\sin 2 \lambda s h 2 \alpha \sin 2 \varphi) \\
+(\operatorname{ch} 2 \alpha \cos 2 \lambda-1) \cos 2 \varphi] R_{i j}^{\mathrm{p}} \gamma_{\mathrm{p}} f_{g} \sum_{h}\left(1-f^{h}\right)
\end{gathered}
$$

La fonction obtenue (33), décrit le lien entre la transformation martenstique et l'évolution de la microstructure au cours de la localisation de la déformation plastique. Elle tient compte des interactions entre les variantes au sein d'un même grain $h$ et $k$, et de l'interaction entre la variante $h$ (le point $M$ ) et les dislocations formées (Fig. 3). 


\section{Application et résultats}

L'alliage de mémoire de forme utilisé $\mathrm{Cu}-\mathrm{Zn}-\mathrm{Al}$ est de type $[3,6,14]$, avec la normale $\vec{n}(0,182,0,669,0,721)$ et de direction $\vec{m}(0,165,0,737,0,655)$ de module de cisaillement $\mu=40 \mathrm{GPa}$ et du coefficient de Poisson $\nu=0,333$, l'amplitude de déformation plastique variant dans le domaine $10^{-4}<\gamma_{\mathrm{p}}<$ quelques $10^{-3}$.

L'échantillon est sollicité en traction, La résolution numérique est obtenue en utilisant une méthode d'optimisation [17], cette méthode consiste à chercher pour chaque $\left(\Sigma_{i j}, T\right)$ les valeurs de la fraction volumique $f^{h}$ qui minimisent le potentiel $\psi$ avec une structure en dislocation donnée (fraction volumique de la zone affectée $f_{\mathrm{p}}=$ const. $\left.=0.01\right)$.

\subsection{Chargement mécanique à $T=20^{\circ}$}

Les résultats des simulations numériques ont conduit à plusieurs résultats importants. Figure 4 : présente l'évolution de la contrainte en fonction de la déformation de transformation, en absence et en présence de l'amplitude de la déformation plastique.

À une amplitude de déformation plastique nulle $\ll \gamma_{\mathrm{p}}=$ 0 », le niveau de contrainte obtenu correspond à la contrainte critique.

À $\gamma_{\mathrm{p}}$ non nulle, le niveau de contrainte diminue. Dans tous les cas la déformation de transformation est de l'ordre $6 \%$, résultats cohérents avec les résultats expérimentaux $[8,18]$.

Sur la figure 5, on représente l'évolution de la contrainte en fonction de la fraction volumique de martensite $f^{h}$.

L'examen de telle courbe montre une évolution identique à celle obtenue sur la figure 4 . À saturation la fraction volumique de la variante $f^{h} \approx 1$.

\subsection{Chargement mécanique à $\neq T^{\circ}$}

Parallèlement des essais mécaniques à différentes températures ont été également étudiés, figure 6 , présente l'évolution de la contrainte en fonction de la déformation de transformation pour $\gamma_{\mathrm{p}}=0$ et $\gamma_{\mathrm{p}}=3 \times 10^{-3}$.

On note une évolution du niveau de contrainte à différentes températures, cet effet est plus marquant à grande déformation dans le domaine où nous nous sommes placés.

Sur la figure 7 , nous représentons la variation de la contrainte en fonction de la température.

On montre une influence de cyclage et des traces de plasticité sur le comportement de l'alliage, résultats cohérents avec les résultats expérimentaux [19].

\subsection{Effet de couplage}

Rappelons-le, notre objectif est de suivre l'évolution de la structure après un certain nombre de cycles, et de

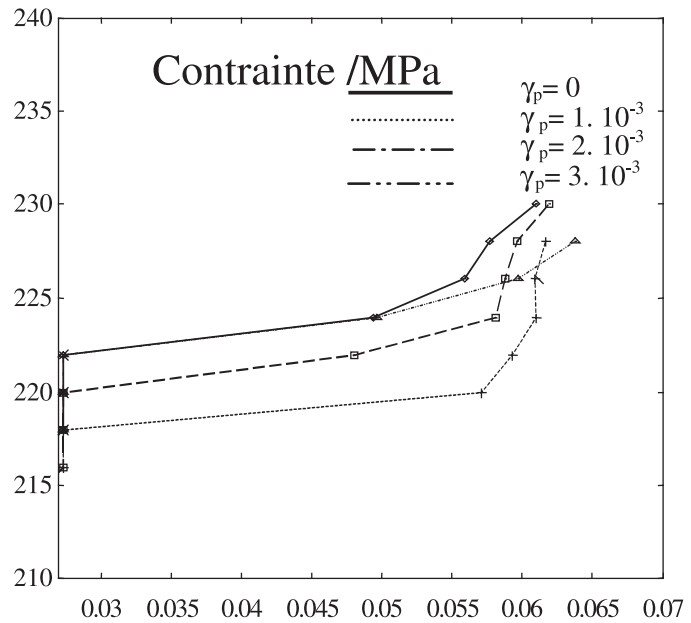

Déformation de transformation

Fig. 4. Évolution de la contrainte en fonction de la déformation de transformation pour différentes amplitudes de déformation plastique.

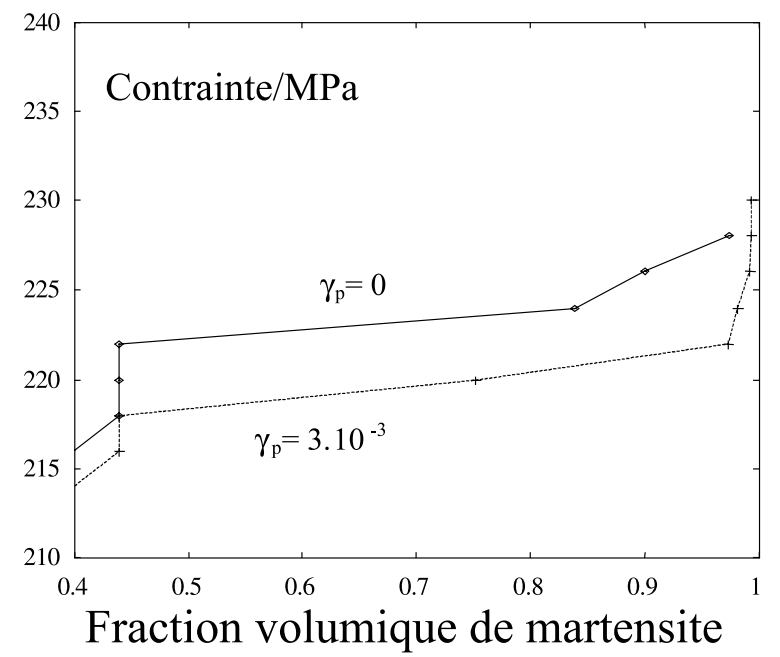

Fig. 5. Évolution de la contrainte en fonction de la fraction volumique de martensite $f^{h}$.

relier les deux phases en évolutions (transformation martensitique et l'évolution microstructurale), ceci peut être déterminé en considérant un critère dit critère de couplage.

Critère de couplage : le couplage est déterminé à partir de la différence des niveaux de contraintes à saturation pour une amplitude de déformation plastique nulle $« \tau_{0} »$, et des contraintes à saturation pour une amplitude de déformation non nulle $« \tau_{\mathrm{s}} »$.

L'évolution de cette différence de contrainte $« \tau_{0}-\tau_{\mathrm{S}}$ » en fonction de l'amplitude de déformation plastique est présentée à la figure 8 .

L'évolution de la différence de contrainte en fonction de la déformation plastique imposée sépare la courbe en deux domaines distincts :

- un domaine « $\mathbf{I} »$ ou la pente contrainte déformation est sensiblement nulle, 


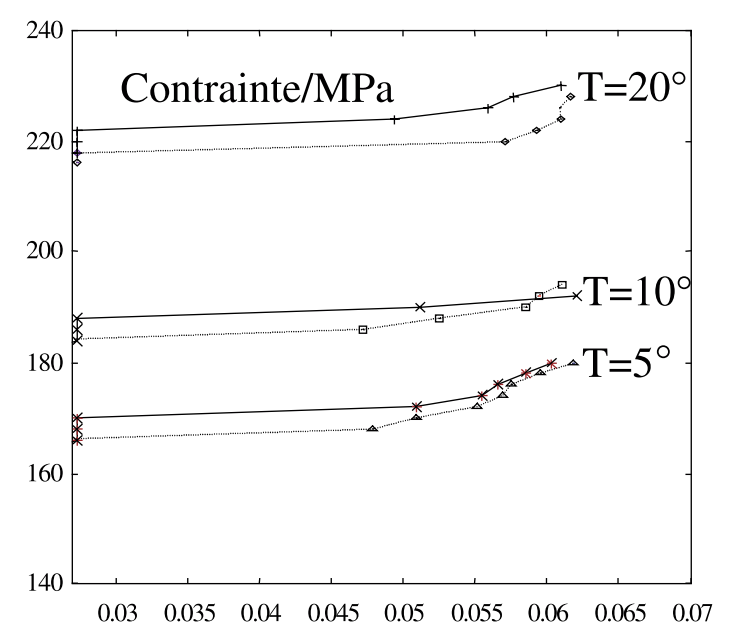

Fig. 6. Évolution de la contrainte - déformation de transformation à différentes températures d'essais pour : $\gamma_{\mathrm{p}}=0$ et $\gamma_{\mathrm{p}}=3 \times 10^{-3}$ (pointillé).

\section{Contrainte/MPa}

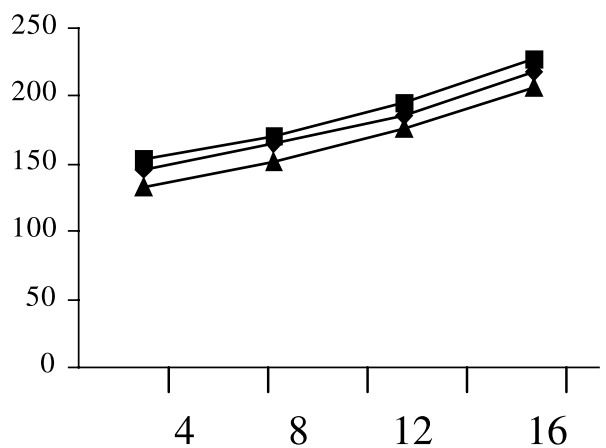

Fig. 7. Évolution de la contrainte en fonction de température à différentes $\gamma_{\mathrm{p}}$.

- un domaine « II » ou la différence de contrainte augmente du façon régulière avec la déformation plastique, phénomène lié à la multiplication et à l'annihilation des dislocations.

\subsection{Charge - décharge à $T=20^{\circ}$}

Dans les alliages à mémoire de forme les chemins de charge et décharge ne sont pas identiques, ceci est due à la forte dissipation d'énergie durant la transformation [20-24].

Pour avoir un maximum d'information à propos de ces essais, nous avons suivi l'évolution de la contrainte au cours de la charge directe « austénite $\rightarrow$ martensite » et le sens inverse la décharge « martensite $\rightarrow$ austénite .

La figure 9 représente ces évolutions pour différentes amplitudes déformations plastiques imposées $\gamma_{\mathrm{p}}=0$, $\gamma_{\mathrm{p}}=1,5 \times 10^{-3}$ et $\gamma_{\mathrm{p}}=2 \times 10^{-3}$.

- À la décharge, quelque soit l'amplitude de déformation plastique imposée le niveau de contrainte diminue, et les cycles d'hystérésis changent de forme au fur est à

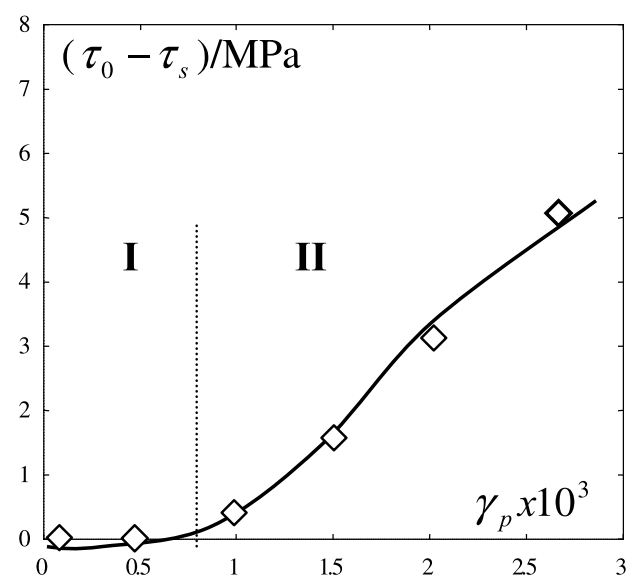

Fig. 8. Évolution de la différence de contrainte $\tau_{0}-\tau_{\mathrm{s}}$ en fonction de l'amplitude déformation plastique.

mesure que le nombre de cycles augmentent, résultats cohérents avec les résultats expérimentaux [14,25].

- Au tout début de la décharge, la martensite n'est pas facile à se décharger des défauts ce qui montre pratiquement sa stabilité, ce phénomène est très accentué lors de la localisation de la déformation plastique.

\section{Discussion}

La modélisation proposée dans ce travail pour un alliage à mémoire de forme en présence de la plasticité donne des réponses physiques très avancées pour ces types des matériaux.

En premier lieu, le modèle suppose une zone de l'échantillon déformée plastiquement, due à l'évolution de la transformation martensitique.

Il montre des modifications des propriétés mécaniques, ces modifications peuvent être expliquées par l'influence de la multiplication et ou annihilations des dislocations.

La diminution de la contrainte critique au cours du cyclage explique partiellement le comportement de l'alliage.

Les cycles d'hystérésis caractéristiques des $A M F s$ ont un caractère loin de pseudoélastique. En effet, au cours de la décharge, il y a une perte de linéarité avant d'atteindre la contrainte nulle. Ceci peut être traduit par un champ des contraintes internes associé aux défauts des dislocations et des précipités.

Conséquence, le piégeage des dislocations ou la localisation de la déformation plastique dans les bandes stabilise alors la phase de martensite. Ce qui peut être expliqué par l'établissement d'un autre mécanisme de déformation.

Dans ce travail nous avons pu coupler entre les deux transformations ; la transformation martensitique et l'évolution de structure. À ce stade, le modèle a pu montrer la différence entre les deux mécanismes envisagés.

Le passage réversible (domaine I) est due à l'orientation préférentielle des variantes de martensite sous l'effet de contrainte, résultats cohérents avec les résultats obtenus [3, 18-22]. 

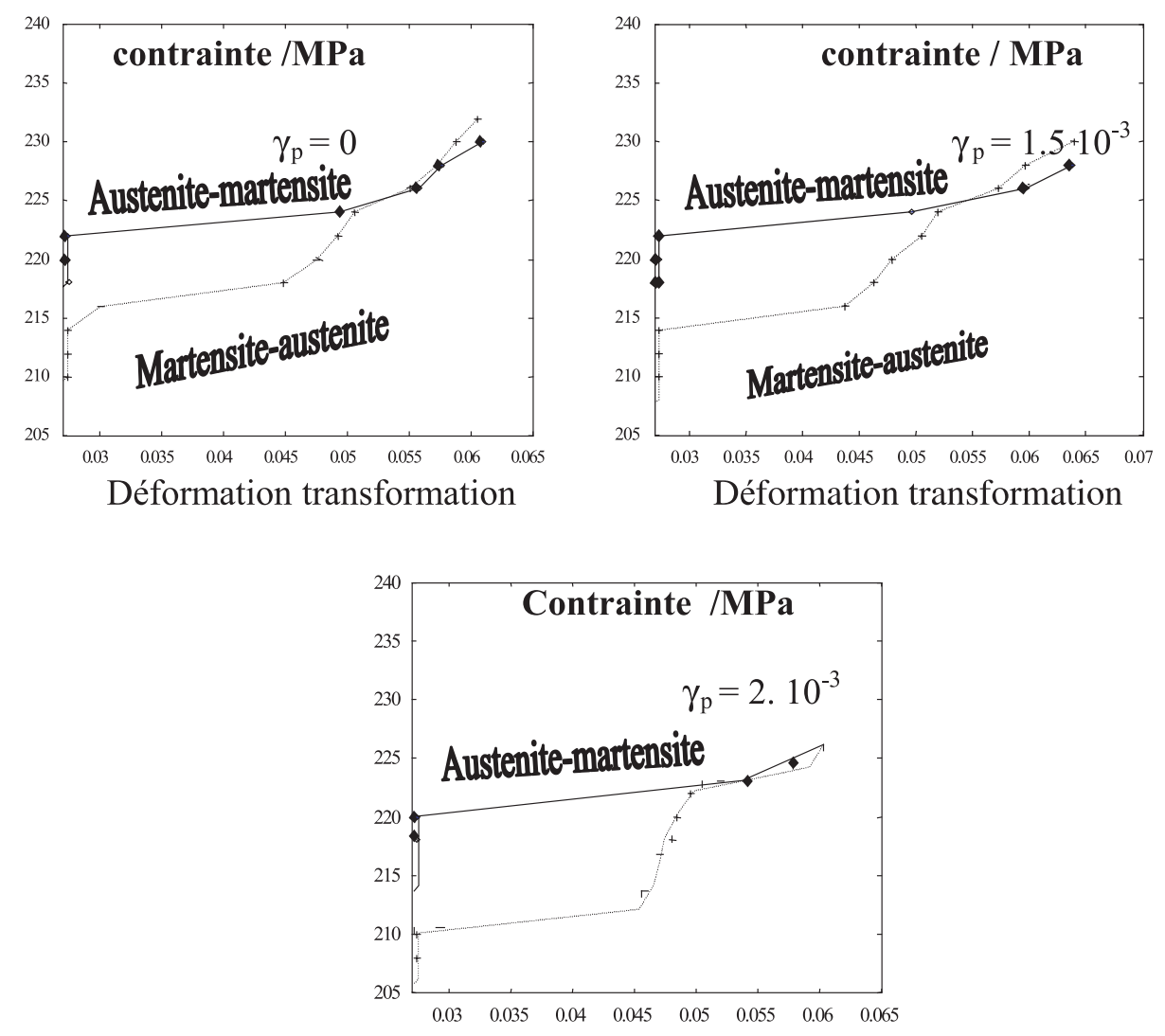

Déformation de transformation

Fig. 9. Boucles dhystérésis « charge-décharge » contrainte-déformation pour différentes amplitudes de déformation plastiques.

À amplitude de déformation plastique non nulle dans le domaine où nous nous sommes situés, l'effet de la plasticité agit sur le niveau de la contrainte critique et sur la déformation de transformation. Dans ce cas la phase de martensite subsiste et devient très sensible à la multiplication des défauts (domaine II).

\section{Conclusion}

Dans ce travail, nous avons développé une approche avec une succession d'équation représentant un potentiel micromécanique métallurgique dans lequel intervient des contributions énergétiques d'origine différente. Cette nouvelle tendance a permis d'enrichir cette modélisation en rapprochant les variables macroscopiques à des évolutions des paramètres microstructuraux.

La modélisation est basée sur l'optimisation de la fonction d'énergie obtenue, qui contient : une énergie chimique, une énergie élastique, une énergie d'échange et une énergie inélastique. Le modèle permet de prédire correctement l'évolution de la structure, telle que la multiplication et l'annihilation des dislocations et leurs interactions.

En se référant à un critère dit critère de couplage, nous avons pu relier les deux phases en transformation. L'effet observé est la manifestation directe des contraintes internes qui représentent une part importante de la contrainte appliquée.

On admet que ces contraintes évoluent peu au début de la transformation et en absence de la déformation plastique c'est-à-dire qu'un éventuel réarrangement de dislocations voire l'annihilation de certaines d'elles n'affectent pas le champ de contraintes de compatibilité. Toutefois, ce phénomène est très accentué dans des zones très riches en dislocations et où des véritables interactions entre les dislocations et la variante se développent dans la direction de glissement. Le changement de structure et en particulier de la phase martensitique entraîne une modification des propriétés mécaniques.

De plus, nous avons montré une dissipation d'énergie durant le retour « élastique », un pourcentage important de cette fraction au tout début de la décharge a été également observé.

Le modèle est capable de suivre à la fois la transformation martensitique et l'évolution de la structure, de décrire les mécanismes mise en jeu au cours de la déformation.

\section{Références}

[1] J. Ortin, A. Planes, Thermodynamics of thermoelastic martensitic transformations, Acta Mettal. 37 (1989) 1433-1441 
[2] A. Ghazali, M.O. Bensalah, S. Agouram, Comportement thermomécanique des AMF mono et polycristallin en transformation martensitique, Int. J. thermal Sci. 10 (1999) 38

[3] S. Leclercq, C. Lexellent, A general macroscopic description of the thermomechanical behavior of SMAs, J. Mech Solids A, 17 (1998) 185-205

[4] Q.P. Sun, K.C. Hwang, Micromechanics modeling for the constitutive behavior of polycristalline SMAs-II study of the individual phenomena, J. Mech. Phys. Solids 41 (1993) 1-17

[5] F. Auricchio, L. Faravelli, SMAs models and applications, CIMNE Barcelona (2001)

[6] E. Patoor, P. Barbe, A. Eberhardt, M. Berveiller, Thermomechanical behavior of SMAs, Arch Mech. 40 (1988) $775-794$

[7] M. Thumann, E. Hrborgen, Thermal and Mechanical fatigue in SMAs, Z. Mettal kde 79 (1988) 119-126

[8] Bo. Zhonghe, C. Dimitris, Lagoudas, Thermomechanical modeling of polycristalline SMAs under cyclic loading, Part III : evolution of plastic strains and two way shape memory effect, Int. J. Engineering Sci. 37 (1999) $1178-1203$

[9] C. Picornell, M. Sade, E. Cesari, Met. Trans A. 25 A (1994) 687-695

[10] D. Rio-Jara, G. Guénin, On the characterization and origin of the dislocations associated with the two way memory effect in Cu-Zn-Al alloys, Scripta Metall. 35 (1987) 109-126

[11] J. Pons, F.C. Lovey, E. Cesari, Electron-microscopy study of dislocation associated with thermal cycling in a CuZnAl shape memory alloys, Acta Metal Mater 38-12 (1990) 2733-2740

[12] M. Benchiheub, S. Belkahla, G. Guénin, Étude de la stabilisation sous contrainte d'un alliage ternaire à mémoire de forme type $\mathrm{Cu}-\mathrm{Zn}-\mathrm{Al}$, Revue de Métallurgie CIT/ Science et Génie des Matériaux (2000) 1471-1479
[13] H. Tobushi, K. Okumara, M. Endo, K. Tanaka, Deformation behavior of TiNi SMA under or stress contolled conditions, Arch Mech. 54 (2002) 75-91

[14] S. Agouram, M.O. Bensalah, A. Ghazali, Micomechanical modelling of the hysteresis behaviour in the thermally induced martensitic phase transitions, Acta Metall. Mater 1 47 (1999) 13-21

[15] J.D. Eschelby, Proc. Roy. Soc. London A 241 (1957)

[16] J.M.C. Li, Electron microscopy and Strength of Crystals., G. Thomas, Washbum (ed.), J. Intersciences. Pub. New York, 1963, pp. 713-776

[17] R. Fletcher, 1969, Optimisation, Académie Press Inc., London

[18] H. Sehitoglu, R. Anderssen, I. Karaman, K. Gall, Cyclic deformation behavior of single crystal NiTi, Materials Sciences and Engineering A 314 (2001) 67-74

[19] C. Bouvet, de l'uniaxial au multiaxial : comportement pseudoélastique des AMF, Thèse de doctorat, Université de Franche-Comté, 2001

[20] M.O. Bensalah, Thèse du $3^{\mathrm{e}}$ cycle, Metz, 1986

[21] J. Muller, H. Xu, On the pseudo-elastic hysteresis, Acta Metall. Mat. 39(3) (1991) 263-271

[22] J. Ortin, F. Preisach, modeling of hysteresis for a pseudoelastic CuZnAl single crystal, J. Applied Physics 71(3) (1992) 1454-1461

[23] Y. Sato, K. Tanaka, Estimation of energy dissipation in alloys due to stress induced martensitic transformation, Res. Mechanica 23 (1988) 381

[24] N. Siredey, A. Eberhardt, Fatigue behaviour of CuAlBe Shape memory single crystals, Materials Sciences and Engineering A 290 (2000) 171-179

[25] C. Dimitris, Lagoudas, Bo. Zhonghe, Thermomechanical modeling of polycristalline SMAs under cyclic loading, Part II material characterization and experimental results for a stable transformation cycle, Int. J. Engineering Sciences 37 (1999) 1141-1173 